\title{
Approximation by Rational Functions in Variable Exponent Morrey-Smirnov Classes
}

\author{
Ahmed Kinj \\ Dept. of Mathematics, Faculty of Science, Tishreen University, Lattakia, Syria \\ Correspondence should be addressed to Ahmed Kinj; a.kinj@tishreen.edu.sy \\ Received 12 December 2021; Accepted 3 January 2022; Published 21 January 2022 \\ Academic Editor: Ding-Xuan Zhou \\ Copyright ( 92022 Ahmed Kinj. This is an open access article distributed under the Creative Commons Attribution License, which \\ permits unrestricted use, distribution, and reproduction in any medium, provided the original work is properly cited. \\ In this work, the direct theorem of approximation theory in variable exponent Morrey-Smirnov classes of analytic functions, \\ defined on a doubly connected domain of the complex plane bounded by two sufficiently smooth curves, is investigated.
}

\section{Introduction}

The classical Morrey spaces were introduced by Morrey in [1] in order to investigate the local behavior of the solutions of elliptic differential equations. Recently, many researchers have investigated function spaces with variable exponents due to their use in several fields of applied mathematics. In particular, function spaces with variable exponents have many applications in areas involving the modeling of electrorheological fluids [2] and image restoration [3]. The variable exponent Morrey spaces introduced in [4] have been studied intensively by various authors (see, for example, [5-7]). The fundamental problem in approximation theory is to express complicated functions by simple functions such as polynomials, wavelets, or rational functions with more useful structures. The theory of approximation is strongly related with the operators and has a considerable number of applications in areas including general marginal distributions such as sampling and machine learning (see [8-10]). Also, the approximation problems in the variable Morrey-Smirnov classes of analytic functions defined on a simply connected domain with a Dini-smooth boundary are proved in [11]. The direct and converse theorems of approximation theory in the classical Morrey-Smirnov classes defined on a simply connected domain with a Dini-smooth boundary were obtained in $[12,13]$. Similar results in the variable exponent Smirnov classes were studied in $[14,15]$.
On a doubly connected domain, the rate of approximation by $p$-Faber-Laurent rational function in Smirnov classes was studied in [16]. Also, the rate of approximation by Faber-Laurent rational function in Smirnov-Orlicz classes and Smirnov classes with variable exponent was obtained in [17]. The approximation property of $(p-\epsilon)$-Faber-Laurent rational functions in the weighted generalized grand Smirnov classes on doubly connected domains is proved in [18].

In the current paper, approximation one direct theorem of approximation theory in variable exponent Morrey-Smirnov classes of analytic functions, defined on a doubly connected domain bounded by two Dini-smooth curves, is obtained.

\section{Preliminaries}

Let $J$ denote the interval $[0,2 \pi]$ or a Jordan rectifiable curve $\Gamma$, and let $\wp$ denote the class of all Lebesgue measurable functions $p():. \Gamma \longrightarrow[1, \infty[$ such that

$$
1<p_{-}=\underset{z \in J}{\operatorname{essinf}} p(z) \leq p^{+}=\underset{z \in J}{\operatorname{esssup}} p(z)<\infty .
$$

We denote by $|J|$ to the Lebesgue measure of $J$. We say $p(.) \in \wp^{\log }$ if there is a constant $c$ such that

$$
\left|p\left(z_{1}\right)-p\left(z_{2}\right)\right| \ln \left(\frac{|J|}{\left|z_{1}-z_{2}\right|}\right) \leq c,
$$


for all $z_{1}, z_{2} \in J$.

For $p(.) \in \wp^{\log }(\Gamma)$, we define $L^{p(.)}(\Gamma)$ the set of all measurable functions $f$ such that

$$
\int_{\Gamma}|f(z)|^{p(z)}|\mathrm{d} z|<\infty .
$$

$L^{p(.)}(\Gamma)$ is a Banach space with respect to the norm

$$
f_{L^{p(.)(\Gamma)}}=\inf \left\{\lambda>0, \int_{\Gamma}\left|\frac{f(z)}{\lambda}\right|^{p(z)}|\mathrm{d} z| \leq 1\right\} .
$$

Let $U$ be a finite simply connected domain with a rectifiable Jordan curve boundary $\Gamma$. Denote $U^{-}=$ext $\Gamma$, $\gamma_{0}=\{w \in \mathbb{C}:|w|=1\}, D=\operatorname{int} \gamma_{0}$, and $D^{-}=\operatorname{ext} \gamma_{0}$. Let $\Gamma_{r}$ be the image of circle $\{w \in \mathbb{C}:|w|=r, 0<r<1\}$ under some conformal mapping of $D$ onto $U$.

For given $1 \leq p<\infty$, we denote by $E^{p}(U)$ the class of analytic functions $f$ in $U$ which satisfies

$$
\int_{\Gamma_{r}}|f(t)|^{p}|\mathrm{~d} t|<\infty
$$

uniformly in $r$.

It is known that every function of class $E^{P}(U)$ has nontangential boundary values almost everywhere on $\Gamma$ and the boundary function belongs to $L^{p}(\Gamma)$ ([19], pp. 438-453).

Also, suppose that $\phi^{*}$ is the conformal mapping of $U^{-}$ onto $D^{-}$normalized by

$$
\phi^{*}(\infty)=\infty, \quad \lim _{z \longrightarrow \infty} \frac{\phi^{*}(z)}{z}>0,
$$

and let $\psi^{*}$ be the inverse of $\phi^{*}$. Let $\phi_{1}^{*}$ be the conformal mapping of $U$ on to $D^{-}$, normalized by

$$
\phi_{1}^{*}(0)=\infty, \lim _{z \longrightarrow 0} z \phi_{1}^{*}(z)>0 .
$$

The inverse mapping of $\phi_{1}^{*}$ will be denoted by $\psi_{1}^{*}$.

The functions $\psi^{*}$ and $\psi_{1}^{*}$ have in some deleted neighborhood of $\infty$ representations

$$
\begin{aligned}
& \psi^{*}(w)=\alpha w+\alpha_{0}+\frac{\alpha_{1}}{w}+\frac{\alpha_{2}}{w^{2}}+\cdots+\frac{\alpha_{k}}{w^{k}}+\cdots, \quad \alpha>0, \\
& \psi_{1}^{*}(w)=\frac{\beta_{1}}{w}+\frac{\beta_{2}}{w^{2}}+\cdots+\frac{\beta_{k}}{w^{k}}+\cdots, \quad \beta_{1}>0 .
\end{aligned}
$$

The functions

$$
\begin{gathered}
\frac{\psi^{* \prime}(w)}{\psi^{*}(w)-z}, \quad z \in U, \\
\frac{\psi_{1}^{*}(w)}{\psi_{1}^{*}(w)-z}, \quad z \in U^{-},
\end{gathered}
$$

are analytic in the domain $D^{-}$, and the following expansions hold [20-23]:

$$
\begin{array}{cl}
\frac{\psi^{* \prime}(w)}{\psi^{*}(w)-z}=\sum_{k=0}^{\infty} \frac{F_{k}(z)}{w^{k+1}}, & z \in U, w \in D^{-}, \\
\frac{\psi_{1}^{*}(w)}{\psi_{1}^{*}(w)-z}=\sum_{k=1}^{\infty}-\frac{\widetilde{F}_{k}(1 / z)}{w^{k+1}}, \quad z \in U^{-}, w \in D^{-},
\end{array}
$$

where $F_{k}(z)$ and $\widetilde{F}_{k}(1 / z)$ are the Faber polynomials of degree $k$ with respect to $z$ and $1 / z$ for the continuums $\bar{U}$ and $\overline{\mathbb{C}} \backslash U$, respectively.

Let $\Gamma$ be a rectifiable Jordan curve in the complex plane with length $\ell$ and let $\Gamma(t, r)=\Gamma \cap B(t, r), t \in \Gamma, r>0$, where $B(t, r)=\{z \in \mathbb{C}:|z-t|<r\}$. The classical Morrey spaces $L^{p, \lambda}(\Gamma)$ for given $0 \leq \lambda \leq 1$ and $1 \leq p<\infty$ are defined as the set of functions $f \in L_{\text {loc }}^{p}(\Gamma)$ such that

$$
\|f\|_{L^{p, \lambda}(\Gamma)}=\sup _{z \in \Gamma 0<r<\ell} r^{-\lambda / p}\|f\|_{L^{p}(\Gamma(t, r))}<\infty .
$$

Let $U=\operatorname{int} \Gamma$, we define the classical Morrey-Smirnov classes $E^{p, \lambda}(U)$ for $0<\lambda \leq 1$ and $1<p<\infty$ as

$$
E^{p, \lambda}(U)=\left\{f \in E^{1}(U), f \in L^{p, \lambda}(\Gamma)\right\} .
$$

We define $\|f\|_{E^{p, \lambda}(U)}:=\|f\|_{L^{p, \lambda}(\Gamma)}$.

Definition 1. Let $p():. \Gamma \longrightarrow[1, \infty[$ be a Lebesgue measurable function satisfying the condition (1), and let $\lambda():. \Gamma \longrightarrow[0,1]$ be a measurable function. We define the variable exponent Morrey spaces $L^{p(.)) \lambda(.)}(\Gamma)$ as the set of Lebesgue measurable functions $f$ defined on $\Gamma$, such that

$$
\mu_{p(.)) \lambda(.)}(f)=\sup _{t \in \Gamma, 0<r \leq \ell} r^{-\lambda(t)} \int_{\Gamma(t, r)}|f(s)|^{p(s)} \mathrm{d} s<\infty .
$$

$L^{p(.)) \lambda(.)}(\Gamma)$ becomes a Banach space with respect to the norm:

$$
\|f\|_{L^{p(.) \lambda(.)(\Gamma)}}=\inf \left\{u>0: \mu_{p(.) \lambda(.)}\left(\frac{f}{u}\right) \leq 1\right\} .
$$

We define the variable exponent Morrey-Smirnov class $E^{p(.)) \lambda(.)}(U)$ as

$$
E^{p(.) \lambda(.)}(U):=\left\{f \in E^{1}(U), f \in L^{p(.) \lambda(.)}(\Gamma)\right\} .
$$

If we define $\|f\|_{E^{p(.))(.)(U)}}:=\|f\|_{\left.L^{p(.)}\right)(.)(\Gamma)}$, the class $E^{p(.) \lambda(.)}(U)$ becomes a Banach space.

Definition 2. A smooth curve $\Gamma$ is called Dini-smooth if

$$
\int_{0}^{\delta} \frac{\Omega(\sigma, s)}{s} \mathrm{~d} s<\infty, \quad \delta>0,
$$

where $\sigma(s)$ is the angle, between the tangent line of $\Gamma$ and the positive real axis expressed as a function of arclength $s$ with the modulus of continuity $\Omega(\sigma, s)$, where 


$$
\Omega(\sigma, s):=\sup _{\left|s_{1}-s_{2}\right| \leq s}\left|\sigma\left(s_{1}\right)-\sigma\left(s_{2}\right)\right|, \quad s>0 .
$$

Definition 3. Let $p():. \gamma_{0} \longrightarrow[1, \infty)$ and $\lambda():. \gamma_{0} \longrightarrow[0,1]$ be measurable functions such that

$$
0 \leq \lambda_{-}:=\underset{t \in \gamma_{0}}{\operatorname{essinf}} \lambda(t) \leq \lambda^{+}:=\underset{t \in \gamma_{0}}{\operatorname{esssup}} p(t)<1
$$

Also, assume that $p(.) \in \wp^{\log }$. For $f \in L^{p(.) \lambda(.)}\left(\gamma_{0}\right)$, we define the operator

$$
\left(v_{h} f\right)(w)=\frac{1}{h} \int_{0}^{h} f\left(w e^{i t}\right) \mathrm{d} t, \quad w \in \gamma_{0}, 0<h<\pi .
$$

The operator $v_{h}$ is bounded linear operator on $L^{p(.)) \lambda(.)}\left(\gamma_{0}\right)$ [24]. Hence, we can define the modulus of smoothness of $f \in L^{p(.)) \lambda(.)}\left(\gamma_{0}\right)$ as

$$
\Omega(f, \delta)_{p(.)) \lambda(.)}=\sup _{0<\mathrm{h} \leq \delta}\left\|f-v_{h} f\right\|_{\left.L^{p(.)}\right) \lambda(.)}\left(\gamma_{0}\right), \quad \delta>0 .
$$

The function $\Omega(f, \delta)_{p(.) \lambda(.)}$ is a continuous, nonnegative, and nondecreasing on $[0, \infty)$ satisfying the following properties for any $f, g \in L^{p(.)) \lambda(.)}\left(\gamma_{0}\right)$ :

$$
\begin{aligned}
& \lim _{\delta \longrightarrow 0} \Omega(f, \delta)_{p(.) \lambda(.)}=0 \text {, } \\
& \Omega(f+g, \delta)_{p(.) \lambda(.)} \leq \Omega(f, \delta)_{p(.) \lambda(.)}+\Omega(g, \delta)_{p(.) \lambda(.)}, \quad \delta>0, \\
& \Omega(f, n \delta)_{p(.)) \lambda(.)} \leq n \Omega(f, \delta)_{p(.) \lambda(.)}, \quad n=1,2, \ldots, \delta>0 .
\end{aligned}
$$

Suppose that $G$ is an arbitrary doubly connected domain in the complex plane $\mathbb{C}$, bounded by two rectifiable Jordan curves $L_{1}$ and $L_{2}$. Without loss of generality, we may assume that the closed curve $L_{2}$ is inside the closed curve $L_{1}$ and $0 \in \operatorname{int} L_{2}$. Let $G_{1}^{0}:=\operatorname{int} L_{1}, G_{1}^{\infty}:=\operatorname{ext} L_{1}, G_{2}^{0}:=\operatorname{int} L_{2}$, and $G_{2}^{\infty}:=\operatorname{ext} L_{2}$.

We denote by $w=\phi(t)\left(w=\phi_{1}(t)\right)$ the conformal mapping of $G_{1}^{\infty}\left(G_{2}^{0}\right)$ onto domain $D^{-}$normalized by the conditions:

$\phi(\infty)=\infty, \lim _{t \longrightarrow \infty} \frac{\phi(t)}{t}>0, \phi_{1}(0)=\infty, \lim _{t \longrightarrow 0} t \phi_{1}(t)>0$.

Moreover, $\psi$ and $\psi_{1}$ will denote the inverse mappings of $\phi$ and $\phi_{1}$, respectively.

The level lines of the domains $G_{1}^{0}$ and $G_{2}^{0}$ are defined for $r, R>1$, by

$$
C_{r}:=\{t:|\phi(t)|=r\}, C_{R}:=\left\{t:\left|\phi_{1}(t)\right|=R\right\} .
$$

The Faber polynomials $F_{k}(t)$ and $\widetilde{F}_{k}(1 / z)$ have the following integral representations [22].

If $t \in \operatorname{int} C_{r}$, then

$$
F_{k}(t)=\frac{1}{2 \pi i} \int_{C_{r}} \frac{[\phi(\xi)]^{k}}{\xi-t} \mathrm{~d} \xi=\frac{1}{2 \pi i} \int_{|w|=r} \frac{\psi^{\prime}(w) w^{k}}{\psi(w)-t} \mathrm{~d} w
$$

If $t \in \operatorname{ext} C_{r}$, then

$$
F_{k}(t)=[\phi(t)]^{k}+\frac{1}{2 \pi i} \int_{C_{r}} \frac{[\phi(\xi)]^{k}}{\xi-t} \mathrm{~d} \xi
$$

If $t \in \operatorname{int} C_{R}$, then

$$
\widetilde{F}_{k}\left(\frac{1}{t}\right)=\left[\phi_{1}(t)\right]^{k}-\frac{1}{2 \pi i} \int_{C_{R}} \frac{\left[\phi_{1}(\xi)\right]^{k}}{\xi-t} \mathrm{~d} \xi .
$$

If $t \in \operatorname{ext} C_{R}$, then

$\widetilde{F}_{k}\left(\frac{1}{t}\right)=-\frac{1}{2 \pi i} \int_{C_{R}} \frac{\left[\phi_{1}(\xi)\right]^{k}}{\xi-t} \mathrm{~d} \xi=-\frac{1}{2 \pi i} \int_{|w|=R} \frac{\psi_{1}^{\prime}(w) w^{k}}{\psi_{1}(w)-t} \mathrm{~d} w$.

If $f(z)$ is an analytic function in the doubly connected domain bounded by the curves $C_{r}$ and $C_{R}$, then

$$
f(t)=\sum_{k=0}^{\infty} a_{k} F_{k}(t)+\sum_{k=1}^{\infty} b_{k} \widetilde{F}_{k}\left(\frac{1}{t}\right)
$$

where

$a_{k}=\frac{1}{2 \pi i} \int_{|w|=r_{1}} \frac{f(\psi(w))}{w^{k+1}} \mathrm{~d} w, \quad 1<r_{1}<r, k=0,1,2, \ldots$,

$b_{k}=\frac{1}{2 \pi i} \int_{|w|=R_{1}} \frac{f\left(\psi_{1}(w)\right)}{w^{k+1}} \mathrm{~d} w, \quad 1<R_{1}<R, k=1,2, \ldots$

Let $L=L_{1} \cup L_{2}^{-}$and let $G$ be a doubly connected domain bounded by $L_{1}$ and $L_{2}$, where $L_{2}$ is in $L_{1}$. We define the variable exponent Morrey-Smirnov classes $E^{p(.)) \lambda(.)}(G)$ as

$$
E^{p(.) \lambda(.)}(G)=\left\{f \in E^{1}(G), f \in L^{p(.)) \lambda(.)}(L)\right\} .
$$

For $f \in E^{p(.) \lambda(.)}(G)$, the norm is defined by

$$
\|f\|_{E^{p(.)) \lambda(.)}(G)}=\|f\|_{L^{p(.) \lambda(.)}(L)} .
$$

Let $U$ be a simply connected domain in the complex plane $\mathbb{C}$, bounded by a rectifiable Jordan curve $\Gamma$, and let $U^{-}$ be the exterior of $\Gamma$. Then, for $f \in L^{1}(\Gamma)$, the functions $f^{+}$ and $f^{-}$defined by

$$
\begin{aligned}
f^{+}(t):=\frac{1}{2 \pi i} \int_{\Gamma} \frac{f(\xi)}{\xi-t} \mathrm{~d} \xi, & t \in U, \\
f^{-}(t) & :=\frac{1}{2 \pi i} \int_{\Gamma} \frac{f(\xi)}{\xi-t} \mathrm{~d} \xi, \quad t \in U^{-} .
\end{aligned}
$$

are analytic in $U$ and $U^{-}$, respectively, $f^{-}(\infty)=0$.

For a given $t \in \Gamma$, the operator $S_{\Gamma}$ defined by

$$
S_{\Gamma}(f)(t):=\lim _{\varepsilon \longrightarrow 0^{+}} \frac{1}{2 \pi i} \int_{\Gamma \cap\{\xi:|\xi-t|>\varepsilon\}} \frac{f(\xi)}{\xi-t} \mathrm{~d} \xi,
$$

is called the Cauchy singular operator.

According to the Privalov theorem [19] if one of the functions $f^{+}$or $f^{-}$has the nontangential limits a.e. on $\Gamma$, then $S_{\Gamma}(f)(t)$ exists a.e. on $\Gamma$ and also the other one has the nontangential limits a.e. on $\Gamma$. Conversely, if $S_{\Gamma}(f)(t)$ exists a.e. on $\Gamma$, then the functions $f^{+}$and $f^{-}$have nontangential limits a.e. on $\Gamma$. In both cases, the following formulae: 


$$
\begin{aligned}
f^{+}(t) & =S_{\Gamma} f(t)+\frac{1}{2} f(t), f^{-}(t)=S_{\Gamma} f(t)-\frac{1}{2} f(t), \\
f(t) & =f^{+}(t)-f^{-}(t),
\end{aligned}
$$

hold a.e. on $\Gamma$.

In Kokilashvili and Meskhi [25], it is proved that, if $\Gamma$ is a Dini-smooth curve, then the operator $S_{\Gamma}$ is bounded on $L^{p(.)) \lambda(.)}(\Gamma)$, i.e., there exists a positive constant $c_{1}$ such the following inequality holds for any $f \in L^{p(.) \lambda(.)}(\Gamma)$ :

$$
\left\|S_{\Gamma}(f)\right\|_{L^{p(.) \lambda(.)(\Gamma)}} \leq c_{1}\|f\|_{L^{p(.) \lambda(.)}(\Gamma)} .
$$

If $L_{1}$ and $L_{2}$ are Dini-smooth curves, then by [26] there are positive constants $c_{2}, c_{3}, c_{4}$, and $c_{5}$ such

$$
c_{2}<\left|\psi^{\prime}(w)\right|<c_{3}, c_{4}<\left|\psi_{1}^{\prime}(w)\right|<c_{5} .
$$

Let $L_{i}(i=1,2)$ be a Dini-smooth curve, we define the following functions $f_{0}:=f \circ \psi$ for $f \in L^{p(.) \lambda(.)}\left(L_{1}\right), p_{0}=$ $p \circ \psi$ and $f_{1}:=f \circ \psi_{1}$ for $f \in L^{p(.) \lambda(.)}\left(L_{2}\right), p_{1}=p \circ \psi_{1}$.

If $f \in L^{p(.)) \lambda(.)}\left(\mathrm{L}_{1}\right)$ and $f \in L^{p(.)) \lambda(.)}\left(\mathrm{L}_{2}\right)$, then by (36), $f_{0} \in L^{p_{0}(.), \lambda(.)}\left(\gamma_{0}\right)$ and $f_{1} \in L^{p_{1}(.) \lambda(.)}\left(\gamma_{0}\right)$. From (34), we get $f_{0}^{-}(\infty)=0, f_{1}^{-}(\infty)=0$ and the following relations hold a.e. on $\gamma_{0}$ :

$$
f_{0}(w)=f_{0}^{+}(w)-f_{0}^{-}(w), f_{1}(w)=f_{1}^{+}(w)-f_{1}^{-}(w) .
$$

Using Theorem 6.1 from [24] and taking into account the proof of a similar result in [20], we deduce the following lemma.

Lemma 1. Let $p():. \gamma_{0} \longrightarrow\left[1, \infty\left[\right.\right.$ and $\lambda():. \gamma_{0} \longrightarrow[0,1]$ be measurable functions. Let $g \in E^{p(.) \lambda(.)}(D)$ with $p(.) \in \wp^{\log }\left(\gamma_{0}\right)$ and $0 \leq \lambda_{-} \leq \lambda^{+}<1$. If $\sum_{k=0}^{n} a_{k}(g) w^{k}$ is the $n-$ th partial sum of the Taylor series of $g$ at the origin, then for any $n=1,2, \ldots$, there is a constant $c_{6}$ such the following estimate:

$$
\left\|g(w)-\sum_{k=0}^{n} a_{k}(g) w^{k}\right\|_{L^{p(.) \lambda(.)}\left(\gamma_{0}\right)} \leq c_{6} \Omega\left(g, \frac{1}{n}\right)_{p(.) \lambda(.)},
$$

holds.

\section{Main Result}

From now on, we will assume that the set of rational functions is dense in the space $E^{p(.)) \lambda(.)}(L)$. Our main result is the following.

Theorem 1. Let $G$ be a finite doubly connected domain with the Dini-smooth boundary, $L=L_{1} \bigcup L_{2}^{-}$, and let $p():. L \longrightarrow\left[1, \infty\left[\right.\right.$, and $\lambda():. L \longrightarrow[0,1] . E^{p(.) \lambda(.)}(L)$ be the variable Morrey-Smirnov space with $p(.) \in \wp^{\log }(L)$ and $0 \leq \lambda_{-}<\lambda^{+}<1$. If $f \in E^{p(.)) \lambda(.)}(G)$, then for every $n \in \mathbb{N}$, there are a rational function $R_{n}(f,$.$) and a constant c_{7}$ such that

$\left\|f-R_{n}(f, .)\right\|_{E^{p(.) \lambda(.)}(G)} \leq c_{7}\left[\Omega\left(f_{0}, \frac{1}{n}\right)_{p_{0}(.) \lambda(.)}+\Omega\left(f_{1}, \frac{1}{n}\right)_{\left.p_{1}(.)\right) \lambda(.)}\right]$.
Proof. Let $f \in E^{p(.,) \lambda(.)}(L)$, then $f_{0} \in E^{p_{0}(.) \lambda(.)}\left(\gamma_{0}\right)$ and $f_{1} \in E^{p_{1}(.) \lambda(.)}\left(\gamma_{0}\right)$.

Putting $\phi(\xi)$ and $\phi_{1}(\xi)$ in place of $w$ in (37), we obtain

$$
\begin{aligned}
& f(\xi)=f_{0}^{+}(\phi(\xi))-f_{0}^{-}(\phi(\xi)), \quad \xi \in L_{1}, \\
& f(\xi)=f_{1}^{+}\left(\phi_{1}(\xi)\right)-f_{1}^{-}\left(\phi_{1}(\xi)\right), \quad \xi \in L_{2} .
\end{aligned}
$$

Let $t \in \operatorname{ext} L_{1}$, then from (24), we have

$$
\sum_{k=0}^{n} a_{k} F_{k}(t)=\sum_{k=0}^{n} a_{k} \phi^{k}(t)+\frac{1}{2 \pi i} \int_{L_{1}} \frac{\sum_{k=0}^{n} a_{k} \phi^{k}(\xi)}{\xi-t} \mathrm{~d} \xi
$$

and using (40), we get

$$
\begin{aligned}
\sum_{k=0}^{n} a_{k} F_{k}(t)= & \sum_{k=0}^{n} a_{k} \phi^{k}(t)+\frac{1}{2 \pi i} \int_{L_{1}} \frac{\sum_{k=0}^{n} a_{k} \phi^{k}(\xi)-f_{0}^{+}(\phi(\xi))}{\xi-t} \mathrm{~d} \xi \\
& +\frac{1}{2 \pi i} \int_{L_{1}} \frac{f(\xi)}{\xi-t} \mathrm{~d} \xi+\frac{1}{2 \pi i} \int_{L_{1}} \frac{f_{0}^{-}(\phi(\xi))}{\xi-t} \mathrm{~d} \xi .
\end{aligned}
$$

Since $f_{0}^{-}(\phi(\xi)) \in E^{p_{0}(.) \lambda(.)}\left(G_{1}^{\infty}\right)$,

$$
\frac{1}{2 \pi i} \int_{L_{1}} \frac{f_{0}^{-}(\phi(\xi))}{\xi-t} \mathrm{~d} \xi=-f_{0}^{-}(\phi(t))
$$

Thus,

$$
\begin{aligned}
\sum_{k=0}^{n} a_{k} F_{k}(t)= & \sum_{k=0}^{n} a_{k} \phi^{k}(t)+\frac{1}{2 \pi i} \int_{L_{1}} \frac{\sum_{k=0}^{n} a_{k} \phi^{k}(\xi)-f_{0}^{+}(\phi(\xi))}{\xi-t} \mathrm{~d} \xi \\
& +\frac{1}{2 \pi i} \int_{L_{1}} \frac{f(\xi)}{\xi-t} \mathrm{~d} \xi-f_{0}^{-}(\phi(t)) .
\end{aligned}
$$

Now, for $t \in \operatorname{ext} L_{2}$, from (27) and (41), we have

$$
\begin{aligned}
\sum_{k=1}^{n} b_{k} \widetilde{F}_{k}\left(\frac{1}{t}\right)= & -\frac{1}{2 \pi i} \int_{L_{2}} \frac{\sum_{k=1}^{n} b_{k} \phi_{1}^{k}(\xi)}{\xi-t} \mathrm{~d} \xi \\
= & \frac{1}{2 \pi i} \int_{L_{2}} \frac{f_{1}^{+}\left(\phi_{1}(\xi)\right)-\sum_{k=1}^{n} b_{k} \phi_{1}^{k}(\xi)}{\xi-t} \mathrm{~d} \xi \\
& -\frac{1}{2 \pi i} \int_{L_{2}} \frac{f(\xi)}{\xi-t} \mathrm{~d} \xi
\end{aligned}
$$

For any $t \in \operatorname{ext} L_{1}$, we have

$$
\frac{1}{2 \pi i} \int_{L_{1}} \frac{f(\xi)}{\xi-t} \mathrm{~d} \xi=\frac{1}{2 \pi i} \int_{L_{2}} \frac{f(\xi)}{\xi-t} \mathrm{~d} \xi
$$

Because ext $L_{1} \subset$ ext $L_{2}$, the relations (45), (46), and (47) are valid for any $t \in \operatorname{ext} L_{1}$, and this gives 


$$
\begin{aligned}
\sum_{k=0}^{n} a_{k} F_{k}(t)+\sum_{k=1}^{n} b_{k} \widetilde{F}_{k}(1 / t)= & =\sum_{k=0}^{n} a_{k} \phi^{k}(t)-f_{0}^{-}(\phi(t))-\frac{1}{2 \pi i} \int_{L_{1}} \frac{f_{0}^{+}(\phi(\xi))-\sum_{k=0}^{n} a_{k} \phi^{k}(\xi)}{\xi-t} \mathrm{~d} \xi \\
& +\frac{1}{2 \pi i} \int_{L_{2}} \frac{f_{1}^{+}\left(\phi_{1}(\xi)\right)-\sum_{k=1}^{n} b_{k} \phi_{1}^{k}(\xi)}{\xi-t} \mathrm{~d} \xi .
\end{aligned}
$$

Taking the limit as $t \longrightarrow z \in L_{1}$ along nontangential path outside $L_{1}$ for almost every $z \in L_{1}$, we get

$$
\begin{aligned}
& f(z)-\left(\sum_{k=0}^{n} a_{k} F_{k}(z)+\sum_{k=1}^{n} b_{k} \widetilde{F}_{k}\left(\frac{1}{z}\right)\right) \\
& =f_{0}^{+}(\phi(z))-\sum_{k=0}^{n} a_{k} \phi^{k}(z)+\frac{1}{2}\left(f_{0}^{+}(\phi(z))-\sum_{k=0}^{n} a_{k} \phi^{k}(z)\right) \\
& \quad+S_{L_{1}}\left(f_{0}^{+}(\phi(z))-\sum_{k=0}^{n} a_{k} \phi^{k}(z)\right)-\frac{1}{2 \pi i} \int_{L_{2}} \frac{f_{1}^{+}\left(\phi_{1}(\xi)\right)-\sum_{k=1}^{n} b_{k} \phi_{1}^{k}(\xi)}{\xi-t} \mathrm{~d} \xi .
\end{aligned}
$$

The rational function $R_{n}(f, z)$ is defined as

By (51), Minkowski's inequality, and (35), we get

$$
R_{n}(f, z)=\sum_{k=0}^{n} a_{k} F_{k}(z)+\sum_{k=1}^{n} b_{k} \widetilde{F}_{k}\left(\frac{1}{z}\right) .
$$

$$
\left\|f-R_{n}(f, .)\right\|_{L^{p(.) \lambda(.)}\left(L_{1}\right)} \leq c_{8}\left\|f_{0}^{+}(w)-\sum_{k=0}^{n} a_{k} w^{k}\right\|_{L^{\left.p_{0}(.)\right)(.)}\left(\gamma_{0}\right)}+c_{9}\left\|f_{1}^{+}(w)-\sum_{k=0}^{n} b_{k} w^{k}\right\|_{L^{p_{1}(.) \lambda(.)}\left(\gamma_{0}\right)}
$$

And from Lemma 1, we obtain Let $t^{\prime} \in \operatorname{int} L_{2}$. From (26) and (41), we get $\left.f-R_{n}(f,).\right)_{L^{p(.)}(.)}\left(L_{1}\right) \leq c_{10}$

$$
\left\{\Omega\left(f_{0}, \frac{1}{n}\right)_{p_{0}(.) \lambda(.)}+\Omega\left(f_{1}, \frac{1}{n}\right)_{\left.p_{1}(.)\right) \lambda(.)}\right\} .
$$

$$
\begin{aligned}
\sum_{k=1}^{n} b_{k} \widetilde{F}_{k}\left(\frac{1}{t^{\prime}}\right)= & \sum_{k=1}^{n} b_{k} \phi_{1}^{k}\left(t^{\prime}\right)-\frac{1}{2 \pi i} \int_{L_{2}} \frac{\sum_{k=1}^{n} b_{k} \phi_{1}^{k}(\xi)}{\xi-t^{\prime}} \mathrm{d} \xi \\
= & \sum_{k=1}^{n} b_{k} \phi_{1}^{k}\left(t^{\prime}\right)-\frac{1}{2 \pi i} \int_{L_{2}} \frac{\sum_{k=1}^{n} b_{k} \phi_{1}^{k}(\xi)-f_{1}^{+}\left(\phi_{1}(\xi)\right)}{\xi-t^{\prime}} \mathrm{d} \xi \\
& -\frac{1}{2 \pi i} \int_{L_{2}} \frac{f(\xi)}{\xi-t^{\prime}} \mathrm{d} \xi-f_{1}^{-}\left(\phi_{1}\left(t^{\prime}\right)\right) .
\end{aligned}
$$


and for any $t^{\prime} \in$ int $L_{1}$, from (24) and (40), we have

$$
\begin{aligned}
\sum_{k=1}^{n} a_{k} F_{k}\left(t^{\prime}\right)= & \frac{1}{2 \pi i} \int_{L_{1}} \frac{\sum_{k=0}^{n} a_{k} \phi^{k}(\xi)}{\xi-t^{\prime}} \mathrm{d} \xi \\
= & \frac{1}{2 \pi i} \int_{L_{1}} \frac{\sum_{k=0}^{n} a_{k} \phi^{k}(\xi)-f_{0}^{+}(\phi(\xi))}{\xi-t^{\prime}} \mathrm{d} \xi \\
& +\frac{1}{2 \pi i} \int_{L_{1}} \frac{f(\xi)}{\xi-t^{\prime}} \mathrm{d} \xi .
\end{aligned}
$$

Since int $L_{2} \subset$ int $L_{1}$, relations (19) and (20) are valid for $t^{\prime} \in \operatorname{int} L_{2}$, and this gives

$$
\begin{aligned}
\sum_{k=0}^{n} a_{k} F_{k}\left(t^{\prime}\right)+\sum_{k=1}^{n} b_{k} \widetilde{F}_{k}\left(1 / t^{\prime}\right)= & \frac{1}{2 \pi i} \int_{L_{1}} \frac{\sum_{k=0}^{n} a_{k} \phi^{k}(\xi)-f_{0}^{+}(\phi(\xi))}{\xi-t^{\prime}} \mathrm{d} \xi-\frac{1}{2 \pi i} \int_{L_{2}} \frac{\sum_{k=1}^{n} b_{k} \phi_{1}^{k}(\xi)-f_{1}^{+}\left(\phi_{1}(\xi)\right)}{\xi-t^{\prime}} \mathrm{d} \xi \\
& -f_{1}^{-}\left(\phi_{1}\left(t^{\prime}\right)\right)+\sum_{k=1}^{n} b_{k} \phi_{1}^{k}\left(t^{\prime}\right) .
\end{aligned}
$$

Taking the limit as $t^{\prime} \longrightarrow z \in L_{2}$ along nontangential path inside $L_{2}$ for almost every $z \in L_{2}$, we get

$$
\begin{aligned}
f(z)-\left(\sum_{k=0}^{n} a_{k} F_{k}(z)+\sum_{k=1}^{n} b_{k} \widetilde{F}_{k}\left(\frac{1}{z}\right)\right)= & f_{1}^{+}\left(\phi_{1}(z)\right)-\frac{1}{2}\left(\sum_{k=1}^{n} b_{k} \phi_{1}^{k}(z)-f_{1}^{+}\left(\phi_{1}(z)\right)\right) \\
& -S_{L_{2}}\left(\sum_{k=1}^{n} b_{k} \phi_{1}^{k}(z)-f_{1}^{+}\left(\phi_{1}(z)\right)\right)-\frac{1}{2 \pi i} \int \frac{\sum_{k=0}^{n} a_{k} \phi^{k}(\xi)-f_{0}^{+}(\phi(\xi))}{\xi-z} \mathrm{~d} \xi
\end{aligned}
$$

Using Minkowski’s inequality and (35), we get

$$
\begin{aligned}
\left\|f-R_{n}(f, .)\right\|_{\left.L^{p(.)}\right)(.)\left(L_{2}\right)} \leq & c_{11}\left\|f_{1}^{+}(w)-\sum_{k=1}^{n} b_{k} w^{k}\right\|_{L^{p_{0}(.) \lambda(.)}\left(\gamma_{0}\right)} \\
& +c_{12}\left\|f_{0}^{+}(w)-\sum_{k=0}^{n} a_{k} w^{k}\right\|_{L^{p_{1}(.) \lambda(.)}\left(\gamma_{0}\right)} .
\end{aligned}
$$

By Lemma 1, we obtain

$$
\begin{aligned}
\left\|f-R_{n}(f, .)\right\|_{L^{p(.) \lambda(.)} \leq} & c_{13} \\
& \cdot\left\{\Omega\left(f_{0}, \frac{1}{n}\right)_{\left.p_{0}(.)\right) \lambda(.)}+\Omega\left(f_{1}, \frac{1}{n}\right)_{\left.p_{1}(.)\right) \lambda(.)}\right\} .
\end{aligned}
$$

From (52) and (58), we obtain

$$
\begin{aligned}
\left\|f-R_{n}(f, .)\right\|_{E^{p(.) \lambda(.)}(G)} \leq & c_{7} \\
& \left\{\Omega\left(f_{0}, \frac{1}{n}\right)_{p_{0}(.) \lambda(.)}+\Omega\left(f_{1}, \frac{1}{n}\right)_{\left.p_{1}(.)\right) \lambda(.)}\right\} .
\end{aligned}
$$

\section{Data Availability}

No data were used to support this study.

\section{Conflicts of Interest}

The author declares that there are no conflicts of interest.

\section{References}

[1] C. Morrey, "On the solutions of quasi- linear elliptic partial differential equations," Transactions of the American Mathematical Society, vol. 43, p. 126, 1938.

[2] M. Růžička, "Electrorheological fluids: modeling and mathematical theory," in Lecture Notes in MathematicsSpringer, Berlin, Germany, 2000.

[3] F. Li, Z. Li, and L. Pi, "Variable exponent functionals in image restoration," Applied Mathematics and Computation, vol. 216, no. 3, pp. 870-882, 2010.

[4] A. Almeida, J. Hasanov, and S. Samko, "Maximal and potential operators in variable exponent Morrey spaces," Georgian Mathematical Journal, vol. 15, no. 2, pp. 195-208, 2008.

[5] T. Ohno, "Continuity properties for logarithmic potentials of functions in Morrey spaces of variable exponent," Hiroshima Mathematical Journal, vol. 38, no. 3, pp. 363-383, 2008. 
[6] V. Kokilashvili and A. Meskhi, "Maximal functions and potentials in variable exponent Morrey spaces," Complex Variables and Elliptic Equations, vol. 55, pp. 923-936, 2010.

[7] M. Izuki, E. Nakai, and S. Sawano, "Function spaces with variable exponents- an introduction," Scientiae Mathematicae Japonicae, vol. 77, no. 2, pp. 187-315, 2014.

[8] H. Karsli, "Asymptotic properties of urysohn type generalized sampling operators," Carpathian Mathematical Publications, vol. 13, no. 3, pp. 631-641, 2021a.

[9] H. Karsli, "On multidimensional Urysohn type generalized sampling operators," Mathematical Foundations of Computing, vol. 4, no. 4, pp. 271-280, 2021 b.

[10] X. Guo, L. X. Li, and Q. Wu, "Modeling interactive components by coordinate kernel polynomial models," Mathematical Foundations of Computing, vol. 3, pp. 263-277, 2020.

[11] S. Jafarov, "Approximation by Faber-Laurent rational functions in variable exponent Morrey spaces," Computational Methods and Function Theory, 2021.

[12] D. M. Israfilov and N. P. Tozman, "Approximation by polynomials in morrey-smirnov classes," East Journal on Approximations, vol. 14, no. 3, pp. 255-269, 2008.

[13] D. M. Israfilov and N. P. Tozman, "Approximation in morreysmirnov classes," Azerbaijan Journal of Mathematics, vol. 1, no. 1, pp. 99-113, 2011.

[14] D. M. Israfilov and A. Testici, "Approximation in Smirnov classes with variable exponent," Complex Variables and Elliptic Equations, vol. 60, no. 9, pp. 1243-1253, 2015.

[15] D. M. Israfilov and A. Testici, "Approximation problems in the Lebesgue spaces with variable exponent," Journal of Mathematical Analysis and Applications, vol. 459, no. 1, pp. 112-123, 2018.

[16] S. Z. Jafarov, "On approximation of functions by p-FaberLaurent rational functions," Complex Variables and Elliptic Equations, vol. 60, no. 3, pp. 416-428, 2015.

[17] S. Z. Jafarov, "Approximation by rational functions in Smirnov-Orlicz classes," Journal of Mathematical Analysis and Applications, vol. 379, no. 2, pp. 870-877, 2011.

[18] A. Testici, "Approximation by rational functions on doubly connected domains in weighted generalized grand Smirnov classes. Ukrainian Mathematical Journal," 2021.

[19] G. Goluzin, "Geometric theory of functions of a complex variable," Translations of Mathematical Monographs, Vol. 26, AMS, , Providence, RI, USA, 1969.

[20] A. Cavus and D. Israfilov, "Approximation by Faber-Laurent rational functions in themean of functions of the class Lp with $1<\mathrm{p}<\infty$," Approx. Theory Appl.vol. 11, no. 1, pp. 105-118, 1995.

[21] P. Duren, Theory of Hp Spaces, Academic Press, New York, NY, USA, 1970.

[22] A. Markushevich, Theory of Analytic Functions, Vol. 2, Izdatelstvo Nauka, , Moscow, Russia, 1968.

[23] P. Suetin, Series of Faber Polynomials, Gordob and Breack Science Publishers, London, UK, 1998.

[24] V. Guliyev, A. Ghorbanalizadeh, and Y. Sawano, "Approximation by trigonometric polynomials in variable exponent Morrey spaces," Analysis and Mathematical Physics, vol. 9, no. 3, pp. 1265-1289, 2019.

[25] V. Kokilashvili and A. Meskhi, "Boundedness of maximal and singular operators in Morrey spaces with variable exponent," Armenian Journal of Mathematics, vol. 1, no. 1, pp. 18-28, 2008.

[26] S. E. Warschawski, "Uber das ranverhalten der Ableitung der Abildunggsfunktion bei Konfermer Abbildung. (German)," Mathematische Zeitschrift, vol. 35, 1932. 